\title{
Kirchenfunk im österreichischen Rundfunk
}

\author{
von Dorothea Winkler
}

Die Intention dieser Studie war es festzustellen, welche Faktoren für die Produktionen des Kirchenfunks im Österreichischen Rundfunk (ORF) maßgebend sind, wobei die Darstellung der speziellen Probleme der beiden kirchlichen Abteilungen des Hörfunks und des Fernsehens - sowie Ansätze zu deren Bewältigung integriert werden sollten.

Im Vergleich mit anderen Abteilungen der Rundfunkanstalt ist es bedeutsam, daß die Hauptträger dieser Sendungen - die kirchlichen Sprecher - trotz ihres echten Einsatzes für die Produktionen als Medienamateure bezeichnet werden müssen. Trotz dieses Engagements blieb der zu wünschende Effekt wegen der Diskrepanz dieser Intentionen und ihrer Realisierungen vielfach aus. Dieses Faktum und das Bestreben, eine Anregung zur Lösung einzelner anstehender Probleme zu geben, setzte den Impuls für die Wahl dieses Themas.

\section{Historischer Hintergrund}

\subsection{Abriß der Geschichte des Kirchenfunks}

Die Arbeitsaufnahme der Österreichischen Radio-Verkehrs AG (RAVAG) am 1. Oktober 1924 fällt weder mit dem Gründungsdatum einer Abteilung „Kirchenfunk" noch mit dem Produktionsbeginn religiöser Sendungen zusammen. Dies lag vor allem an den politischen und kulturpolitischen Spannungen zwischen den Parteien, die ihre Vertrauensmänner auf den nach Proporz verteilten Posten der RAVAG hatten. Um diesen Auseinandersetzungen zu begegnen, hatte sich die Direktion der RAVAG zu einem Neutralismus entschlossen, weshalb die Behandlung religiöser und politischer Themen grundsätzlich ausgeschlossen war.

Die ersten Sendungen mit religiösem Hintergrund wurden in den Jahren 1926 und 1927 gesendet. Die erste Übertragung einer katholischen Messe fand 1929 statt.

1931 wurde Rudolf Henz, der schon längere Zeit Mitglied des RAVAG-Beirates als Vertreter des Volksbundes der Katholiken und des Österreichischen Radio-Bundes war, zum Direktor der wissenschaftlichen Abteilung ernannt. Nach seinem Eintritt in die RAVAG war ein starkes Ansteigen der religiösen Sendungen zu bemerken, so fand 1931 auch die erste evangelische Sendung statt. Die Errichtung einer eigenen Kirchenfunkabteilung wurde von Rudolf Henz stets abgelehnt, da er die wesentliche christliche Durchdringung nicht in den kirchlichen Sendungen, sondern in der allgemeinen Tendenz des Programmes sah².

Mit Ausbruch des Krieges setzte eine Pause in der Produktion religiöser Sendungen ein, die bis Kriegsende andauerte.

Dr. phil. Dorothea Winkler arbeitete 1969 bis 1977 im Amt für Rundfunk, Film und Fernsehen der Evangelischen Kirche in Österreich, 1979/80 als Lokalredakteurin im Faber-Verlag Krems und ist z. Zt. Stipendiatin des Lutherischen Weltbundes in der BR Deutschland mit dem Ziel einer Fachausbildung in kirchlicher Medienarbeit. 
1945 gab es in Österreich - entsprechend der Zoneneinteilung der Besatzungsmächte - vier Sendergruppen, die kirchliche Sendungen brachten. Teilweise wurde an den Sonntagen in den Jahren 1946 und 1947 von jeder Sendergruppe ein anderer Gottesdienst übertragen. Eine turnusmäßige Übertragung wurde erst am 4. November 1947 anläßlich einer Rundfunk-Enquete in Salzburg beschlossen.

Das Fortbestehen kirchlicher Sendungen nach 1945 war zu einem großen Teil auf das persönliche Engagement einzelner bedeutender Persönlichkeiten des Rundfunks zurückführen. Hauptsächlich war es dem bis Ende 1957 das Amt des Programmdirektors bekleidenden Rudolf Henz zu verdanken, daß die Belange der Kirchen im Rundfunk weiterhin gewahrt wurden, vor allem in der Übergangsphase 1954 von den vier Sendergruppen auf die drei Programme des Österreichischen Rundfunks. Das Proporzdenken, das zu dieser Zeit im Rundfunk vorherrschte, machte auch den kirchlichen Sendungen zu schaffen, die der Proporzhälfte der Östereichischen Volkspartei zugerechnet wurden. Die Steuerung der Sendungen besorgte eine Zentrale von Wien aus, da eine echte Mitarbeit der Bundesländer weitgehend fehlte. Deshalb kamen auch die Sprecher größtenteils aus dem Raum um Wien.

Die Organisation der Sendungen war zu diesem Zeitpunkt für den katholischen Bereich sehr einfach. Die Wahl der Kirchen für Gottesdienstübertragungen wurde in erster Linie durch die Qualität ihres Chores bestimmt, da diese Übertragungen hauptsächlich als kirchenmusikalische Veranstaltungen gesehen wurden. Für die weiteren kirchlichen Sendungen erhielt die 1950 innerhalb der Katholischen Aktion gegründete „Arbeitsgemeinschaft für Rundfunkfragen“ Sendezeiten zugeteilt, für die sie Sprecher auswählte.

Die Zusammenarbeit mit der Evangelischen Kirche lief über den 1953 gegründeten „Evangelischen Rundfunkdienst“, der die Vergabe von Sendezeiten an einzelne Sprecher durchführte, wobei die Termine diözesan aufgeteilt wurden.

Bereits 1959 plante Programmdirektor Alfons Übelhör, eine Abteilung „Kirchenfunk" ins Leben zu rufen. Nach zweijährigen Verhandlungen zwischen den Parteien und den Kirchen über die Errichtung einer Kirchenfunkstelle einigte man sich vorerst grundsätzlich, jedoch nicht sofort, über die personelle Besetzung dieser Position. Ab 1. Juli 1961 wurde Ing. Walter Karlberger, der vor diesem Zeitpunkt Werbeleiter und Organisationsreferent des Niederösterreichischen Pressehauses war, mit dieser Sonderabteilung im Hörfunk betraut.

Eine der wichtigsten Aufgaben der Abteilung war die Erstellung eines Budgets, andererseits der Aufbau einer gesamtösterreichischen Kirchenfunkabteilung, für die noch keinerlei Konzeption vorlag. Weiterhin mußten Organisation und Produktion der Sendungen, die in verschiedenen Händen lagen, koordiniert werden. Die Problematik, ein Angebot für Fernstehende zu schaffen, neben der Befriedigung des Intensivsegmentes, war ebenfalls zu lösen. Eine Revision der verwendeten Sendeformen und Inhalte war nötig. Langsam begann man kirchliche Verkündigung von kirchlicher Information und darunter wieder Nachricht und Kommentar zu unterscheiden. Der Aufbau einer Abteilung Kirchenfunk war nach zwei Jahren mit der Erstellung eines lückenlosen Diözesanproporzes grundsätzlich erledigt. In den einzelnen Landesstudios des Österreichischen Rundfunks mußten Referenten für den Kirchenfunk bestellt werden. 
1963 erfolgte eine Neuor dnung der mit den Medien befaßten Stellen der Katholischen Kirche. Nach der Verabschiedung des „Dekretes über die sozialen Kommunikationsmittel“ (Inter mirifica) vom 4. Dezember 1963 wurde im Sinne von Kapitel II, Absatz 21 und 22 (darin wird für jedes Land die Bildung einer Zentralstelle für Presse, Film, Rundfunk und Fernsehen vorgesehen) die Errichtung eines solchen Gremiums notwendig. Am 25. September 1965 fand die konstituierende Sitzung des Zentrums für Funk, Film und Fernsehen statt, dem nach einem von der Österreichischen Bischofskonferenz genehmigten Statut die praktische Arbeit der kirchlichen Sendungen, aber auch die Pflege der internationalen Verbindungen vorbehalten blieb. Der größere Teil der Diözesen löste nach der Gründung des Zentrums seine Arbeitsgemeinschaften für Rundfunk und Fernsehen auf und lie $B$ deren Aufgaben in das Zentrum übergehen.

Regelmäßige Planungssitzungen fanden statt. Die neuen diözesanen Hörfunkkommissionen beschäftigten sich mit der Sprecherauswahl und der Qualität der Texte, wobei sich immer wieder Schwierigkeiten aus dem Fehlen von medientheoretisch und journalistisch versierten Fachleuten ergaben. Sprecherseminare für die einzelnen Sendekategorien wurden abgehalten und ab 1972 jährlich eine Studientagung organisiert, die Gelegenheit zu prinzipiellen Gesprächen geben sollte.

Auf evangelischer Seite wurde die Medienarbeit erst 1967 neu geordnet, als die 6. Generalsynode der Evangelischen Kirche in ihrer 4. Session am 7. April 1967 ihre Zustimmung gab, ein „Amt für Rundfunk, Film und Fernsehen der Evangelischen Kirche in Österreich" zu errichten. Ihm oblag es, den Hörfunkdienst beim Österreichischen Rundfunk und den Kirchenfunkabteilungen des Auslandes zu vertreten, Sprecher auszubilden, die Tagungen des Hörfunkdienstes vorzubereiten und zwischenkirchliche Kontakte zur Produktion gemeinsamer Sendungen herzustellen.

Am 1. Oktober 1967 trat die große Programmreform des sich nun als „ORF“ bezeichnenden Österreichischen Rundfunks - unter Leitung des ersten Generalintendanten Gerd Bacher - in Kraft. Sie brachte eine totale Neustrukturierung der drei Programme. Ein drittes, vollwertiges Programm wurde geschaffen, weshalb nicht nur neue Sendereihen konzipiert werden mußten; der durch Ö 3 hervorgerufene Wandel des Präsentationsstils hatte natürlich auch Rückwirkungen auf die kirchlichen Sendungen.

$\mathrm{Zu}$ diesem Zeitpunkt wagte die an und für sich nicht besonders risikofreudige Abteilung Kirchenfunk ihr größtes Experiment, den Start einer Sendereihe, an der alle in Österreich anerkannten Religionsgemeinschaften beteiligt sind, die „Ökumenische Morgenfeier". Durch diese neue Sendereihe lernten sich die Vertreter der einzelnen Kirchen in zunehmendem Maße kennen, wodurch sich die zwischenkirchliche Zusammenarbeit verbesserte. Die damals in einer gewissen Stagnation befindliche Abteilung erhielt neuen Auftrieb. Die kirchlichen Sendungen im Hörfunk wie im Fernsehen nahmen zu, weshalb die Sparte „Kirchenfunk" des Hörfunks im Herbst 1972 auch zu einer „Hauptabteilung“ aufgewertet wurde.

\subsection{Abriß der Geschichte des Kirchenfernsehens}

Am 1. Januar 1957 begann der regelmäßige Fernsehbetrieb. Gelegentlich wurden Sendungen, die kirchliche Ereignisse zum Inhalt hatten, ausgestrahlt, doch erst 1961 setzte eine gewisse Regelmäßigkeit ein. 
1963 erschien es der Österreichischen Bischofskonferenz notwendig, eine eigene kirchliche Abteilung im Fernsehen anzuregen. Direktor Gerhard Freund zögerte die Anstellung des von der Bischofskonferenz für diese Position nominierten Ernst Niesner immer wieder hinaus. Schließlich erhielt Niesner einen mit 1 . Oktober 1965 beginnenden Ein-Jahresvertrag. Damit erfolgte jedoch noch keine Institutionalisierung des Kirchenfernsehens. Er wurde dem Fernsehdirektor direkt unterstellt und erhielt kein eigenes Budget. Neben der Verbesserung der Fernsehansprachen versuchte sich Ernst Niesner 1967 in einem neuen Metier, dem Fernsehspiel. Seiner Meinung nach konnte dem Image der Kirche durch die offene und klare Auseinandersetzung mit Problemen im Fernsehspiel mehr genützt werden als durch viele kleine Sendungen. In den nächsten Jahren wurden - zum Teil mit großem Erfolg - fünf Fernsehspiele produziert. Im September 1975 übernahm er die Leitung einer anderen Abteilung; die Nachfolge in der Leitung der Fernseh-Abteilung Kirche trat Anton Fellner an.

Zur Planung des allgemeinen Programmes und zur Befassung mit grundsätzlichen Fragen des Mediums wurde von der Katholischen Kirche im Zuge der Gründung des Zentrums für Massenkommunikation am 25. September 1965 eine eigene Fernsehkommission geschaffen.

Die Planung der evangelischen Sendungen erfolgte nach Absprache mit dem Vorsitzenden des „Evangelischen Rundfunkdienstes“, Georg Traar, und seit seinem Bestehen am 7. April 1967 mit dem „Amt für Rundfunk, Film und Fernsehen der Evangelischen Kirche in Österreich". Im Zuge der Rundfunkreform 1967 wurde die „Kirche“ zu einem Hauptreferat erklärt und dem Fernsehdirektor direkt unterstellt. Schließlich entwickelte sie sich zu einer eigenen Abteilung mit einem eigenen Budget und einer gewissen Selbständigkeit.

\section{Rechtliche Position des Kirchenfunks und Kirchenfernsehens im ORF-Gefüge}

2.1 Verankerung im Rundfunkgesetz 1974 und in den Allgemeinen Richtlinien für die Programmgestaltung

Im wesentlichen wird der Programmauftrag des Österreichischen Rundfunks im $\S 2$ des Rundfunkgesetzes 1974 geregelt; Absatz 3 des \$ 2 enthält eine Regelung, die für die rechtliche Position des Kirchenfunks und Kirchenfernsehens im ORF von inhaltlicher Bedeutung ist. Es heißt hier: „Bei der Planung des Gesamtprogramms ist die Bedeutung der gesetzlich anerkannten Kirchen und Religionsgesellschaften angemessen zu berücksichtigen."

Natürlich sind die im Rundfunkgesetz enthaltenen allgemeinen Programmgrundsätze wie z.B. die Verpflichtung zur Objektivität bei der Information der Allgemeinheit, die Abgrenzung der Meinungskommentare von den analytischen Sachkommentaren, die Bedeutung der Grundsätze der österreichischen Verfassungsordnung wie die Bedachtnahme auf die bundesstaatliche Gliederung auch für jene Programmteile bindend, die in weiterem Sinn von den Kirchen und Religionsgesellschaften gestaltet werden oder über diese berichten. Weiter besteht auch kein Zweifel, daß die Qualitätsbestimmung des $§ 2$, Absatz 4 des Rundfunkgesetzes - daß vor allem die künstlerischen, volksbildenden und staatspolitischen Sendungen des Hörfunks und Fernsehens sich durch hohes Niveau auszuzeichnen haben - in gleichem Maße auf die Sendungen der Hauptabteilung Kirchenfunk und der Stabsstelle Kirche zutrifft. 
Eine inhaltliche Interpretation des Absatz 3 des $\S 2$ ist in den vom Kuratorium des ORF 1976 erlassenen allgemeinen Richtlinien für die Programmgestaltung, Programmerstellung und Programmkoordinierung in Hörfunk und Fernsehen enthalten, wo es heißt: „Bei der Erfüllung der Verpflichtung gemäß $\S 2$ Abs. 3 RFG (angemessene Berücksichtigung der Bedeutung der gesetzlich anerkannten Kirchen und Religionsgesellschaften) sind nicht nur die durch diese Institutionen gesetzten Ereignisse in ihrer gesellschaftspolitischen Relevanz, sondern auch die Glaubensinhalte dieser Kirchen und Religionsgesellschaften zu berücksichtigen."

Diese vom höchsten Organ des ORF beschlossene Interpretation des gesetzlichen Programmauftrages stellt sicher, daß dieser nicht nur formal und protokollarisch, sondern inhaltlich zu verstehen ist. Die beiden Bestimmungen - der Gesetzesauftrag und die Programmrichtlinien - stellen somit die inhaltliche Basis für die Arbeit der Hauptabteilung Kirchenfunk und der Stabsstelle Kirche im Österreichischen Rundfunk dar.

\subsection{Mitwirkung von Vertretern der gesetzlich anerkannten Kirchen und Reli-} gionsgesellschaften in den Organen des Unternehmens

Seit dem Inkrafttreten des Rundfunkgesetzes 1974 wirken die Kirchen und Religionsgesellschaften in zweifacher Weise in den Organen des Unternehmens mit. Einmal stellen sie zwei von 35 Mitgliedern der Hörer- und Sehervertretung, wobei je eines von der „römisch-katholischen Kirche“ bzw. der „evangelischen Kirche“ bestellt wird. In dieser Funktion haben sie ein Mitwirkungs- und Mitbeschlußrecht vor allem an den Programmempfehlungen dieses Organs.

Zum anderen sind die Kirchen und Religionsgesellschaften durch einen Vertreter im Kuratorium, im höchsten Organ des Unternehmens, präsent, wodurch sie an sehr entscheidenden Leistungs- und Genehmigungsbeschlüssen dieses Organs Anteil haben. Die Wahl dieses Mitglieds obliegt der Hörer- und Sehervertretung, die sechs der insgesamt dreißig Mitglieder des Kuratoriums zu bestellen hat.

\section{Darstellung der Produktionsabteilungen „Kirche" im ORF}

\subsection{Hörfunk: Hauptabteilung Kirchenfunk ( $\mathrm{H} \mathrm{4)}$}

Die Aufgaben der $\mathrm{H} 4$, die für sämtliche Kirchenfunksendungen verantwortlich zeichnet, wurden durch die Organisationsanweisung des Generalintendanten vom 15. Januar 1968 geregelt:

„1. die Koordination aller Hörfunkprogramme in der Sparte Kirchenfunk;

2. die Planung aller Hörfunksendungen der Sparte Kirchenfunk;

3. die Produktion von Sendungen der Sparte Kirchenfunk, die von gesamtösterreichischem Interesse der Religionsgemschaften sind, in allen Hörfunkprogrammen;

4. die Koordination aller Fragen der Kirchenmusik in Zusammenhang mit den Gottesdienstübertragungen und anderen Kirchenfunksendungen;

5. die Koordination aller Produktionen des Hörfunks und des Fernsehens in der Sparte Kirchenfunk ${ }^{3}$;

6. die ständige Kontaktnahme mit den in Österreich gesetzlich anerkannten Religionsgemeinschaften in allen Programmfragen; 
7. die Vertretung des Hörfunkprogrammdirektors in allen Kirchenfunkfragen nach dessen besonderem Auftrag."

Dieses Papier muß als Grundkonzept zur Führung der Abteilung angesehen werden. Die Interpretation der Grundsatzthesen liegt jedoch ausschließlich beim Hauptabteilungsleiter des Kirchenfunks. Bisher kam es zu keiner schriftlichen Abmachung, z. B. über die Vergabe von Sendezeiten an die einzelnen Religionsgemeinschaften oder hinsichtlich der Inhalte der Sendungen. Die Verpflichtung zur Produktion aller konfessionellen Programme geht nur andeutungsweise aus den Punkten 1., 2., und 6. hervor. Außerdem ist für die von den Kirchen gegründeten Kontaktstellen nur ein indirektes Mandat zur Mitarbeit (Punkt 6) abzuleiten.

Die Sendetermine werden von der Hauptabteilung Kirchenfunk in Zusammenarbeit mit der Programmabteilung erstellt. Der Katholischen Kirche, als größter Religionsgemeinschaft Österreichs, steht der Hauptteil der Sendezeit zur Verfügung. Das Ausmaß der Sendezeit, das den anderen Kirchen und Religionsgemeinschaften eingeräumt wird, richtet sich prozentual nach der Zahl ihrer Mitglieder, wobei die kleinen Kirchen über ihren Anspruch hinaus, also überproportional, berücksichtigt werden.

Die Hauptabteilung Kirchenfunk im ORF besteht aus zwei hauptamtlichen Angestellten: dem Spartenleiter und seiner Sekretärin. In den einzelnen Landesstudios ist das Referat Kirchenfunk in der Regel dem Referenten der Sparten Kultur, Wissenschaft oder Literatur zugeteilt. Die Verfasser von Manuskripten, Sprecher und weitere programmgestaltende Personen sind sowohl in der Hauptabteilung Kirchenfunk wie in den Landesstudios freie Mitarbeiter.

Die Referenten der $\mathrm{H} 4$ in den Landesstudios unterstehen personell dem jeweiligen Landesintendanten, in Programmfragen des Kirchenfunks jedoch dem Leiter der $\mathrm{H} 4$ in Wien. Neben der Herstellung eigener Sendungen obliegt der Hauptabteilung auch die Zuteilung der Sendezeiten an die einzelnen Landesstudios. Darüber hinaus können die Kirchenfunk-Referenten der Landesstudios in eigener Verantwortung Sendungen bzw. Sendereihen für ihr Lokalprogramm gestalten, das in die Kompetenz der Landesintendanten fällt. Zur Besprechung der Halbjahresprogramme finden zweimal jährlich Spartensitzungen statt, die der Planung und der Besprechung aktueller Probleme dienen.

Als Partner stehen der Hauptabteilung Kirchenfunk auf katholischer Seite die Hörfunkkommission des „Katholischen Zentrums für Massenkommunikation“ und auf evangelischer Seite das „Amt für Rundfunk, Film und Fernsehen“ gegenüber. Die $\mathrm{H} 4$ ist diesen kirchlichen Gremien aber weder verantwortlich noch ist sie an deren Weisungen gebunden.

Den Spartenreferenten stehen in jeder katholischen Diözese eine diözesane Hörfunkkommission zur Verfügung. Die evangelische Kirche besitzt in jeder ihrer Superintendenzen einen Rundfunkbeauftragten, dessen Aufgabe unter anderem in der Unterstützung des Spartenreferenten des Landesstudios besteht.

Von seiten des ORF gibt es für einige Sendereihen ständige Gestalter und Betreuer für andere ständige Teams. Für die Reihe „Ökumenische Morgenfeier“ wurde zusätzlich der „Ökumenische Arbeitskreis des ORF“ geschaffen, der sich in zunehmendem Maße auch mit anderen ökumenischen Projekten des Rundfunks befasst. 


\subsection{Fernsehen: Stabsstelle Kirche (FZ 3) ${ }^{4}$}

Als „Stabsstelle“ ist die FZ 3 den beiden Fernseh-Intendanten (Programm FS 1 und Programm FS 2) unterstellt, womit die durch das Rundfunkgesetz verlangte Berücksichtgigung der Kirchen im Gesamtprogramm besser bewerkstelligt werden soll. In der Organisationsanweisung vom 11. März 1976 werden die Aufgaben der Dienststelle wie folgt erläutert:

„Mitwirkung an der Planung aller Fernsehsendungen zur angemessenen Berücksichtigung der Bedeutung der gesetzlich anerkannten Kirchen und Religionsgesellschaften.

Ständige Kontaktnahme mit den in Österreich gesetzlich anerkannten Kirchen und Religionsgesellschaften in allen Fernsehprogrammfragen."

Die Organisationsanweisung, die erstmalig eine schriftlich festgehaltene Verankerung der Mitarbeit der FZ 3 in Sendungen anderer Abteilungen vorsieht, hat einen großen Mangel aufzuweisen. In ihr ist kein Auftrag zur Programmproduktion enthalten. In der Praxis ist FZ 3 jedoch dazu verpflichtet, da sie die ihr zukommenden Programmtermine auszufüllen hat.

Analog zur $\mathrm{H} 4$ besteht die Stabsstelle Kirche (FZ 3) aus zwei Angestellten, dem Leiter der Dienststelle und seiner Sekretärin. Auch in der FZ 3 sind sämtliche zusätzlichen an der Produktion der Sendungen beteiligten Personen freie Mitarbeiter. Seit das Fernsehen in zunehmendem Maße mit Regionalbeiträgen arbeitet, sind die Kirchenfunk-Referenten der einzelnen Landesstudios damit befaßt, auch die Belange des Fernsehens ihrer Sparte in ihrem Landesstudio wahrzunehmen. Das bedeutet keine Verpflichtung, jeden Beitrag selbst zu gestalten, wohl aber die, die Verantwortung dafür zu übernehmen.

Auf kirchlicher Seite wird die Stabsstelle Kirche durch die Fernsehkommission des „Katholischen Zentrums für Masenkommunikation“ unterstützt, wie evangelischerseits durch das „Amt für Rundfunk, Film und Fernsehen der Evangelischen Kirche in Österreich". Den Spartenreferenten in den Landesstudios stehen - parallel zu der Situation im Hörfunk - auf katholischer Seite die diözesanen Vertretungen der Fernsehkommission, auf der evangelischen Seite die Rundfunkbeauftragten der Superintendenzen zur Verfügung.

In Pressekritiken wurden Produkte dieser Stabsstelle gelegentlich als Belangsendungen [der Kirchen] bezeichnet. Dies sind sie jedoch weder aus der Sicht der Kirchen noch nach dem Rundfunkgesetz noch vom Selbstverständnis des Leiters dieser Stabsstelle. Belangsendungen (nach österreichischem Sprachgebrauch; vergleichbar wären etwa die Parteien-Wahlsendungen in der BR Deutschland) sind als Sendungen zu definieren, für die der ORF zwar die Sendefunktion hat, aber keine qualitative Verantwortung für Form und Inhalt trägt. Dies trifft bei den Produktionen der $\mathrm{H} 4$ und $\mathrm{FZ} 3$ nicht zu, da die Abteilungen die volle Verantwortung für die formale Gestaltung tragen. Für den Inhalt ist, da es sich bei den meisten kirchlichen Sendungen um einen Meinungskommentar handelt, der Verfasser bzw. Sprecher selbst verantwortlich. Doch auch für diesen Personenkreis hat der Wortlaut von Absatz 2, Abteilung III des Informationsstatutes des ORF Geltung:

„Jeder Mitarbeiter trägt die persönliche Verantwortung für die Einhaltung des Rundfunkgesetzes sowie der vorliegenden Richtlinien des Informationsstatutes 
und ist seinem Vorgesetzten gegenüber unmittelbar verantwortlich. In seiner Verantwortung ist er gedeckt, wenn er die Zustimmung seiner unmittelbaren Vorgesetzten besitzt.“

Das bedeutet, daß der jeweilige kirchliche Sprecher zwar nicht den theologischen Inhalt betreffend, aber hinsichtlich der Einhaltung des Rundfunkgesetzes und des Informationsstatutes dem Spartenreferenten bzw. dem Spartenleiter verantwortlich ist. Da Belangsendungen aber in bezug auf ihren Inhalt weder den Bestimmungen des Rundfunkgesetzes noch denen des Informationsstatutes des ORF unterliegen, können die Vorwürfe, es handle sich bei den Produktionen der $\mathrm{H} 4$ oder FZ 3 um solche, mit Recht zurückgewiesen werden.

Die Bedeutung der Abteilungen geht über den engen Rahmen der Produktion kirchlicher Programme weit hinaus. Diesem Anspruch wird derzeit oft nur intentional Rechnung getragen; da auf beiden Seiten - bei den Kirchen wie beim ORF Grundsatzüberlegungen zum Problem „Verkündigung in den Medien“ fehlen, stehen die beiden Produktionsabteilungen „Kirche“ in einem bisher noch nicht abgeschlossenen Lernprozeß.

\subsection{Die Bedeutung der Abteilungen „Kirche“ im ORF}

Die Kirchen begründen ihren Anspruch auf Sendezeit aber nicht nur mit dem Hinweis auf das Rundfunkgesetz 1974, in dem ihnen als pluralistischen Gruppierungen innerhalb des Staates eine entsprechende Berücksichtigung garantiert wurde. Maßgebend ist vielmehr ein Standpunkt der Kirchen, den Heinz Linnerz so definiert: „Heißt es nicht - und durchaus begründet von ihrem Selbstverständnis her - : die Kirche hat nicht eine Meinung neben der anderen einzubringen, schon gar nicht einen Interessenstandpunkt, sondern Letztverbindliches, den 'Weg, die Wahrheit und das Leben'?"5

Hiermit billigt sich die Kirche eine Bedeutung zu, die sie in eine problematische Lage zwängt. Auf der einen Seite steht sie - aus ihrer Sicht - mit ihrem Anspruch weit über allen anderen gesellschaftlichen Gruppierungen, auf der anderen muß sie jedoch weiterhin Bittsteller um eine - verglichen mit anderen Programmsparten - geringe Sendezeit bleiben. Die Frage, welche Position einer Abteilung „Kirche“ im Hörfunk oder im Fernsehen zukommt, kann nur im Bewußtsein dieses Spannungsfeldes gestellt werden.

Die Problematik beginnt bereits mit dem Titel der Abteilungen „Kirchenfunk“ und „Kirche“. Dies läßt den Schluß zu, daß sich die Abteilungen in ihren Sendungen lediglich mit der „Kirche“ als Körperschaft auseinanderzusetzen haben. Das trifft aber sicher nicht zu. In der Organisationsanweisung der $\mathrm{H} 4$ wird von „Hörfunkprogrammen“ und „Hörfunksendungen“ der Sparte Kirchenfunk und nicht von „Kirchenfunkprogrammen“ und „Kirchenfunksendungen“ gesprochen. Eine Einstellung der derzeit sehr auf die Kirchen ausgerichteten Sendungen und die Konzeption völlig neuer Sendetypen wäre nach diesem Text vollkommen gerechtfertigt.

Anders ist die Lage bei der Stabstelle im Fernsehen. In der sie betreffenden Organisationsanweisung wird von der „Mitwirkung an der Planung aller Fernsehsendungen zur angemessenen Berücksichtigung der Bedeutung der gesetzlich anerkannten Kirchen und Religionsgesellschaften" gesprochen. Eine weiter gefaßte Inter- 
pretation der Aufgaben in Richtung „Religion“ und „Theologie“ ist in diesem Wortlaut nicht enthalten. Hier wäre es nun tatsächlich möglich, ausschließlich auf kirchliche Interessen ausgerichtete Programme einzukaufen oder zu produzieren. Eine Korrektur dieser Organisationsanweisung scheint unumgänglich, muß doch auch für deren Programmierung die Aussage von Heinz Linnerz Geltung haben: „Der Kirchenfunk ist keine Funkveranstaltung der Kirche oder im Sinne der Kirchen, sondern er ist eine Veranstaltung des Funks für seine Hörer."

Das bedeutet, daß der Rundfunk Sendungen veranstaltet, um den kirchlichen, theologischen und religiösen Bedürfnissen seiner Hörer gerecht zu werden und nicht bestimmten Interessen der Kirchen.

Die Existenz der Abteilungen steht und fällt mit der tatsächlichen Relevanz des christlichen Bewußtseins für unsere Gesellschaft. So lange ein repräsentativer Teil der Bevölkerung ein Interesse an der Vermittlung dieses Gedankengutes bekennt, ist die Existenz der Abteilungen $\mathrm{H} 4$ und FZ 3 zur Vermittlung von Religion, Theologie und Stellungnahmen der Kirchen berechtigt. Eine Selbstdarstellung der Kirchen allein liegt nicht im Interesse der Hörer und Seher und darf Hauptzweck der Sendungen sicherlich nicht sein.

Natürlich ist es Aufgabe des Rundfunks, über die Kirchen als gesellschaftsbildenden Kräften des Landes zu berichten und offizielle Stellungnahmen zu Tagesaktualitäten einzuholen. Dies fiele aber hauptsächlich in den Kompetenzbereich der Informationsabteilungen des Hörfunks und Fernsehens.

Die Tatsache, daß sich sowohl die $\mathrm{H} 4$ als auch die FZ 3 einerseits mit der Vermittlung von Religion und Theologie, andererseits aber auch mit der Berichterstattung über die Kirchen beschäftigt, haben sie und ihre Mitarbeiter des öfteren im komplizierte Situationen gebracht. Es kann nicht Aufgabe ein- und derselben Abteilung sein, Verkündigungssendungen unter Mithilfe der kirchlichen Amtsträger, der kirchlichen Organisationen - eben der Kirche - zu produzieren, andererseits aber über eben diese die Öffentlichkeit kritisch und objektiv zu informieren. Die notwendige Distanzierung von diesem Anspruch müßte die Einstellung der Produktion von kirchlichen Nachrichten- und Informationssendungen seitens der $\mathbf{H} 4$ und FZ 3 folgen sowie eine Übernahme der Verantwortung für diese Sendereihen seitens der Informationsabteilungen des ORF. Eine Mitarbeit kirchlicher Fachleute ist jedoch - da für diverse Beiträge spezielle Kenntnisse erforderlich sind - weiterhin unbedingt notwendig.

Was soll nun aber Kirchenfunk sein?

Heinz Linnerz definiert ihn so: „Der Kirchenfunk ist kein Instrument der Verkündung, auch nicht schlicht umgekehrt der Prügel, mit dem „Kritik an der Kirche“ eingebleut wird. Er ist zuerst und insbesondere wie alle anderen Sparten des Programms darauf aus, Öffentlichkeit herzustellen, wobei seine spezifische Aufgabe darin besteht, das öffentliche Gespräch zwischen Kirche und Gesellschaft zu ermöglichen, und zwar in allen Lebensbereichen, von den Fragen der öffentlichen Moral bis zu den letzten Fragen des Menschen. Dabei vertritt er gegenüber dieser Öffentlichkeit nicht ein kirchliches Interesse, sondern er bezieht Kirche und Gesellschaft aufeinander. Er muß sich dabei auch an die Spiel- und Stilregeln halten, die heute für das öffentliche Gespräch gelten oder gelten sollen, also: Unabhängigkeit, Sachbezogenheit, Argumentation, Fairneß." ${ }^{\text {" }}$ 
Eine so verstandene Mitarbeit in der Kirchenfunkabteilung könnte ein Korrektiv für die Kirchen darstellen, an dem die Relevanz ihrer Aussagen für den Menschen von heute überprüfbar wäre. In diesem Sinne könnte der Kirchenfunk den Kirchen wirklich dienen. Walter Schricker meint dazu: „Kirchenfunk ist die Chance einer millionenfachen Präsenz der Kirche, einer Präsenz, die freilich nicht aus Weihrauch bestehen kann, sondern in einer ehrlichen Auseinandersetzung geschehen muß zuerst mit dem eigenen Standpunkt der Kirche.“"

Erkennt man den Abteilungen diese Funktionen zu, müßte dies u.a. eine Umbenennung, etwa in „Kirche und Gesellschaft“, zur Folge haben. Die Zusammenarbeit von diesem Anspruch her neu zu überdenken, wäre jedoch nicht nur Sache des $\mathrm{ORF}$, sondern auch die der führenden kirchlichen Gremien.

Eine kirchliche Mitarbeit darf sich aber nicht nur auf die der $\mathrm{H} 4$ und FZ 3 zugeteilten Sendetermine beschränken. Kirchliches Engagement am ORF kann deshalb nicht im Sitz und Stimme im Kuratorium und im Hörer- und Seherbeirat beginnen und in der Teilnahme an Redaktionssitzungen für die einzelnen „Kirchensendungen"aufhören. Sie muß an der Basis der Produktion aller Programmbereiche wirken und sich dort durch kollegiale Hilfestellungen auf verschiedenen Gebieten eine Existenzberechtigung schaffen. Dies wird in der Kontaktherstellung zu den Verantwortlichen der einzelnen Programmbereiche und zu den mit der Produktion von Sendungen Beschäftigten geschehen müssen. Unterstützung bei der Realisierung von Projekten, Versorgung mit ausreichenden Informationen, Themenvorschläge, vielleicht auch Bereitstellung von Manuskripten, Treatments und Drehbüchern auf der Basis des freien Angebotes sollten folgen.

\section{Gesamtproduktion der ORF Hauptabteilung Kirchenfunk im Jabr 1976}

Im Jahr 1976 wurden von der Hauptabteilung Kirchenfunk 1501 Sendungen (davon 1476 Reihensendungen und 25 Einzelsendungen) mit einer Gesamtsendezeit von 18.122 Minuten oder 302 Stunden und 2 Minuten produziert und gesendet. Da die Programme Ö 1 (6.343 Stunden), Ö R (3.850 Stunden) und Ö 3 (8.089 Stunden) zusammen 18.282 Stunden produzierten, betrug der Gesamtprogrammteil der $\mathrm{H} 4$ 1976 1,65 Prozent.

\section{Gesamtproduktion der Stabstellen Kirche im Jabr 1976}

Der Gesamtbeitrag der FZ 3 zu den beiden Fernsehprogrammen des ORF betrug im Jahr 1976166 Sendungen (davon 124 Reihensendungen und 42 Einzelsendungen) mit 2.715 Sendeminuten oder 45 Stunden und 15 Minuten.

\section{Problematisierung kircblicher Verkündigung in den elektronischen Medien}

\subsection{Allgemeine Vorbemerkung}

Die Literatur, die Ergebnisse der Kommunikationsforschung auf die Produktion kirchlicher Sendungen im Hörfunk und Fernsehen bezieht, ist sehr zahlreich. Aufgabe dieses Abschnitts ist es nicht, einen Überblick über diese Medienliteratur zu geben, sondern spezielle Probleme der Zusammenarbeit zwischen ORF und Kirche darzustellen und sie mit Hilfe der aktuellen Fachliteratur zu interpretieren. 


\subsection{Klärung des Begriffes „Verkündigung“}

Die Klärung dieses Begriffs wird in der publizistikwissenschaftlichen Fachliteratur (i.e.S.) nicht weiter erörtert, da diese Frage den Kirchen und ihren Vertretern zuzuweisen ist. Die Frage der Beziehung zwischen Information und Verkündigung, die des öfteren gestellt wird, betrifft jedoch nicht nur die Verkündigung in den Medien, sondern die gesamte Verkündigungspraxis der Kirchen. Eine allgemeine Anerkennung der von Stefan Rehrl geäußerten Ansicht wäre wünschenswert: „Information und Verkündigung schließen einander nicht aus, vielmehr ist es so, daß die beste Verkündigung jene ist, die einen hohen Informationsgehalt aufweist." ${ }^{" 9}$

In der Kirche liegt des öfteren noch der Irrtum vor, daß Glaube, der die Verkündigung ja trägt, und Information zwei inhomogene Größen sind; zeigt sich in der Medienarbeit die Notwendigkeit einer konkreten Entscheidung, wird sie eher zugunsten des Glaubens als für die Information gefällt. Hans Jürgen Schultz zeigt, daß diese strenge Trennung sowohl in der Begriffswelt als auch in der praktischen Arbeit nicht notwendig ist: „Man kann, meine ich, in einem reichlichen Ausmaß über die Kirche, über ihre Geschichte, über ihre verschiedenen Ausprägungen in der Ökumene, über ihre Theologie, über aktuelle Vorgänge in ihr etc. informieren. Man kann auch über den Glauben informieren. Aber noch besser: man kann glaubend informieren. Glaube ist dann weniger Thema als vielmehr eine Voraussetzung, ein Impuls, ja vielleicht sogar ein Stil der Information." ${ }^{\text {"10 }}$

Und gerade diese Betonung des eigenen Standpunktes, der dem Hörer Tatsachen anbietet, die Urteilsbildung aber ihm zubilligt, ist der Inhalt einer kommunikativen, theologischen reflektierten Information, die der Rezipient von der Kirche heutzutage erwartet. Definiert der Sprecher diesen „bestimmten Standpunkt“ als sein Bekenntnis, so geschieht Information aus Bekenntnis.

„Information aus Bekenntnis aber heraus, nenne ich Verkündigung. ${ }^{\text {"11 }}$ Verkündigung als Information hat sich in der herkömmlichen Predigtpraxis wie in der öffentlichen Rede in den Medien weitgehend noch nicht durchgesetzt. Es scheint überflüssig, eine spezielle Rundfunkhomiletik zu entwickeln, jedoch eine nach Situationen und Zielgruppen unterschiedliche allgemeine Verkündigungsdidaktik. Die Lösung dieses Problems wird nur in Zusammenarbeit von Theologen, Medienwissenschaftlern und Medienfachleuten zu finden sein. Ihr Ergebnis sollte die kirchliche Arbeit in und mit den Medien wesentlich beeinflussen und daher auch reflexive Auswirkungen auf die kirchliche Predigtpraxis haben.

\subsection{Die Situation des kirchlichen Rundfunksprechers}

In den durch den Titel als Sendungen der $\mathrm{H} 4$ und FZ 3 gekennzeichneten Produktionen erscheinen sie dem Rezipienten als Vertreter einer Gruppe, deren Auftrag sie erfüllen. Sie sind verpflichtet, Sachfragen der Kirche aufgrund der Dogmen und höchsten Entscheidungen sachlich zu behandeln. Auf der anderen Seite werden die Sendungen mit dem Namen des jeweiligen Sprechers gekennzeichnet, doch „Träger der Aussage ist ... letztlich die Gruppe selbst, die in den technischen Medien ein Mittel ihrer öffentlichen Wirksamkeit erkannt hat. Die Rolle ihres Sprechers über die Massenmedien erschöpft sich in der Aufgabe des Multiplikators der für die Gruppe verbindlichen Meinung." ${ }^{\text {"2 }}$ 
Deshalb erfolgt eine Kontrolle der Einhaltung der Regeln sowohl durch die Institution, ihre Vertreter wie durch das kirchliche Intensivsegment der Hörer und Seher.

Die Hauptspannung der Sprecher resultiert aus der Diskrepanz zwischen Inhalt und Präsentation. Wird der Inhalt zwar nach persönlichem Ermessen formuliert, so wurde doch eben gezeigt, daß er sich nach den Lehrmeinungen der offiziellen Kirchen zu richten hat. Die Präsentation muß aber persönlich und individuell erfolgen. Diese Spannung führt zu einem aus der Psychologie bekannten „Rollenkonflikt ${ }^{\star 13}$, der für die Sprecher Frustrationsgefühle mit sich bringen kann. Es darf angenommen werden, daß sich eine große Anzahl der Sprecher aus dieser Situation dadurch befreit, daß sie sich in eine Sprache flüchtet, die Walter Jens als „,befremdlich homiletisches Esperanto ${ }^{\text {«14 }}$ bezeichnet. Doch dadurch wird der Rollenkonflikt nur an der Oberfläche, jedoch nicht in seinen Ursachen gelöst.

Werden Fragen in Zusammenhang mit der offiziellen Lehrmeinung der Kirchen aufgegriffen, so besteht nur die Möglichkeit einer deutlichen Abgrenzung zwischen Information durch den Sprecher von der persönlichen Interpretation. Zudem entspräche die Betonung der persönlichen Aussage nicht nur der Realität, sondern auch der Interpretation kirchlicher Sendungen als Meinungskommentar dem österreichischem Rundfunkgesetz 1974.

\subsection{Die Innerkirchlichkeit mancher Sendungen}

Aus der Themenwahl, der Präsentation, wie aus der in kirchlichen Sendungen üblichen Sprache ergibt sich ein Mißverständnis zwischen Kirche und Öffentlichkeit. Der dabei entstehende Regelkreis konnte bis dato noch nicht unterbrochen werden.

Kirchliche Sendungen werden von einer breiten Öffentlichkeit als Äußerung einer für diese Gesellschaft relevanten Gruppe akzeptiert. „Wenn sie ssch dabei aber auf ihren 'göttlichen Auftrag 'beruft und damit den Anspruch allgemeiner Verbindlichkeit erhebt, dann setzt sie in diesem Augenblick innerhalb der Gesellschaft sozusagen einen säkularen Selbstschutzmechanismus in Gang. "15

Dies erklärt auch Publikationen in der österreichischen Presse, die zwar eine Überrepräsentation von Kirchenberichten im Gesamtprogramm des ORF ankreiden, ihre Wurzel jedoch in diesem „säkularen Selbstschutzmechanismus“ haben. Die damit angegriffene Kirche verteidigt nun ihre Position, und wie Lorey fortführt: sie „wird ... immer mehr bemüht sein, solche Sendungen zu produzieren oder zu initiieren, die ihre eigene Bedeutung unterstreichen." ${ }^{\text {"16 }}$

Resultat dieses Fehlverhaltens der Kirche ist eine weitere Ablehnung durch die Öffentlichkeit. Sie wird, so paradox dies klingen mag, zum Ärgernis, weil sie sich nicht zum "Ärgernis ihrer Botschaft" bekennt. Um aber weiter ihren Platz in den Medien behalten zu können, wird sie ,in ihrem öffentlichen Reden ein Bewußtsein ... konsolidieren, das dem Ärgernis ihrer Botschaft gar nicht entspricht. Denn in diesem Bewußtsein ist das Ärgerliche an der christlichen Botschaft längst entschärft beziehungsweise zur Harmlosigkeit umgedeutet." ${ }^{\text {"17 }}$

Als Folge dieser selbst auferlegten Konsolidierung mit der Öffentlichkeit wird die Kirche nun nicht nur ihrer Botschaft nicht gerecht, sondern büßt an Glaubwürdigkeit und damit auch an Interesse der Öffentlichkeit ein. Das erkennend, zieht sie sich weiter in ihre eigenen Bastionen - „Ghetto-Sendungen und GhettoProblematik“ zurück. Der Regelkreis ist geschlossen. 
Ein Aufheben der Innerkirchlichkeit mancher Sendungen kann nur durch eine inhaltliche und sprachliche Öffnung der Kirchen und folglich der kirchlichen Rundfunksprecher im Blick auf die Rezipienten erfolgen. Als Konsequenz dürfen sich alle an diesem Kommunikationsprozess Beteiligten nicht als autarke Größen, sondern als einander bedingende Konstitutiven eines größeren Ganzen sehen.

\subsection{Das Publikumsinteresse}

Laut der ORF-Hörfunkstudie $1976^{18}$ interessieren sich 30 Prozent der erwachsenen Bevölkerung Österreichs für religiöse Sendungen. 23 Prozent zeigten kein Interesse, und 47 Prozent machen dazu keine Angaben ${ }^{19}$. Detaillierte Erwartungen der Rezipienten beider elektronischen Medien wurden in dieser Studie nicht erhoben, wohl aber in der des Jahres 1972, die im Auftrag des Instituts für Moraltheologie an der Universität Salzburg durchgeführt wurde ${ }^{20}$. Als Ergebnis dieser Untersuchung kann der Wunsch der Rezipienten nach stärkerer Lebensnähe der kirchlichen Sendungen bezeichnet werden. Die Erwartungen des Publikums konzentrieren sich auf Information, die aus dem Glauben resultiert. Deutlich abgeschlagen ist die „Darstellung des Glaubens". Aus den Einzelwerten wird deutlich, daß das Publikum eine Zuwendung der Kirche zu den öffentlichen und individuellen Fragen der Gesellschaft - gegenüber der Manifestierung der Institution in den üblichen Ausdrucksformen - den Vorzug gibt. Bei einer Analyse dieser Ergebnisse je nach dem Grad der Einstellung zur Kirche zeigt es sich, daß bei allen Einstellungstypen die unter dem Begriff „Information aus dem Glauben“ subsumierten Erwartungen dominieren.

Die Tendenz zur Information läßt sich auch aus der Hörfunkstudie 1976 entnehmen, bei der sich herausstellte, daß der Anteil der Hörer, die religiösen Sendungen „leicht positiv“ oder „positiv“ gegenüber stehen, relativ hoch mit dem Interesse für kritische Sozialsendungen, Bewältigung von Alltagsproblemen, politischen Sendungen und Informationssendungen sowie Wissenschafts- und Bildungssendungen korreliert $^{21}$.

Die Berücksichtigung der angeführten Ergebnisse müßte sowohl formale wie inhaltliche Konsequenzen für die Gestaltung der Sendungen der H 4 und der FZ 3 mit sich bringen.

\subsection{Die Sprache kirchlicher Sendungen}

Kirchliche Sprache in den Medien muß sich im Kontext mit der in anderen Sendungen verwendeten Sprache sehen und dabei die von der sonstigen kirchlichen Verkündigung unterschiedliche Rezipientensituation betrachten. Die kirchliche Fachsprache und die darin verwendeten Begriffe sind oft von dem gültigen Bezugssystem der Alltagssprache weit entfernt und werden von den Rezipienten großteils nicht verstanden. Der Exklusivitätscharakter dieser Sprache, der weite mögliche Publikumskreise ausschließt, widerspricht jedoch der auf die Allgemeinheit ausgerichteten Intention kirchlicher Verkündigung. Die Problematik, der sich der Rundfunkprediger ausgesetzt sieht, beschreibt Hans Jürgen Schultz: „Es geht bei der Verkündigung im Rundfunk um nichts Geringeres als um eine völlig neue Bewährung der Weltlichkeit unseres Redens von Gott ${ }^{22}$.

Dieses „weltliche“ Reden von Gott steht in dem Anspruch, zwar „in der Öffentlichkeit“, aber „mit jedem einzeln“ zu sprechen. Die Voraussetzung des einen ist die 
Kenntnis der politischen, psychologischen und sozialen Verhältnisse der Rezipienten und eine entsprechende Ausrichtung der Texte; die des anderen aber die Aussage - auf der genannten Basis - in einer persönlichen, direkten Sprach- und Redeweise. Sicher kann es nicht darum gehen, eine theologische leere Sprache zu propagieren, sondern darum, den Kontakt zu dem Rezipienten durch eine Sprache herzustellen, in der der Sprecher auch bereit ist, von sich selber zu sprechen. Er sollte zeigen, daß die aufgestellten Postulate auch für ihn keine Selbstverständlichkeiten bedeuten und er sich, in dem Versuch, diesen gerecht zu werden, genauso von der Botschaft betroffen fühlt wie die Rezipienten seiner Sendungen. Durch dieses persönliche, fast private Reden, soll in ein Gespräch mit dem Partner, dem Rezipienten, übergeführt werden. Ein Heraustreten aus „seiner Rolle" und ein Kommentar zum eigenen Text - geäußert in der Art eines „beiseite Sprechens“ könnten dem Redner wirkungsvolle Unterstützungsmöglichkeiten bieten und ihn so für den Zuhörer „transparent" und "fühlbar" werden lassen.

\subsection{Die Visualisierung}

\subsection{Die Grundproblematik}

Diese liegt in der Tatsache, daß das zu „Transportierende“ - der religiöse Inhalt dem Medium zuwiderläuft. Diese spezielle Problematik wird von der FZ 3 in vielen Sendungen dadurch gelöst, daß man über das mit dem Objektiv festzuhaltende Wirken Gottes in der „organisierten Religion“, also über die für die Religion zuständigen, fachlich qualifizierten, verantwortlichen Institutionen - die Kirchen - berichtet. Man zeigt ihre Tätigkeiten, ihre Struktur, ihre Probleme: ihren Dienst an der Gesellschaft. Dadurch bleibt ein Großteil der Sendungen den kirchlichen Belangen verhaftet und dringt nicht in die religiösen Belange vor. Sicher ist von der Abteilung FZ 3 Kirche nicht die Lösung jenes Problemes zu verlangen, das Franz Zöchbauer mit den Worten festhielt: „Allzu lange war man gewohnt, mit dem Wort zu verkündigen. Es wurde eine Theologie des Wortes begründet. Wir haben aber noch keine Theologie des Bildes."23

Von seiten der Kirchen, die sich für die Sprache des Wortes, aber nicht für die des Bildes zuständig fühlen, besteht eine gewisse distanziert-abwartende Haltung. Diese distanzierte Haltung, die ausschlaggebend für das Nichtvorhandensein einer Theologie des Bildes ist, muß schon deshalb aufgehoben werden, da eine Theologie des Bildes in den Fernsehproduktionen enthalten ist, jedoch hauptsächlich von Medienpraktikern und -journalisten, kaum unter Mitarbeit von Theologen, verantwortlich eingesetzt wird. Hans-Dieter Bastian beschreibt diese Situation:

„Kirchenleitende Amtsträger stellen bevorzugte Konfliktpersonen dar, die man semantisch gerne ins Zwielicht rückt. Der Titel 'Oberhirt' im entsprechenden Medienkontext attackiert das schläfrige Bewußtsein aller Kirchenschafe. Eine Kamerafahrt an leeren Bänken entlang, eventuell mit unterlegtem Choral, spricht Bände. Alle Informationen sind hier hochgradie Meinungsträger, auch dann, wenn die Medienabsicht Kritik verspricht ... So existiert, abseits von Kanzeln und Kathedern, eine wichtige Trivialtheologie mit leistungsstarken Kontaktkanälen, denen zur vollen Kommunikation nur dies fehlt, da $B$ die 'offizielle' Theologie sie kennt."24

Will die Kirche den Zuschauern nicht lediglich eine Privattheologie der Gestalter und damit eine von ihr nicht gewollte und unter Umständen auch nicht verantwortbare Theologie zukommen lassen, wird sie sich mit diesen Fragen befassen und 
eigene Verantwortliche auf dieses Gebiet heranbilden müssen, die in Zusammenarbeit mit den von der Filmästhetik her qualifizierten Mediengestaltern Inhalte dann mit der intendierten Aussage umsetzen können.

\subsection{Gedanken zur Visualisierung religiöser Inhalte}

Da es sich dabei zumeist um abstrakte Inhalte handelt, ergeben sich bei der Umsetzung ins Fernsehen größere Schwierigkeiten, weil eine direkte Wortinterpretation mittels Bildsprache nicht adäquat erscheint. Hingegen scheint die Auflösung religiöser Begriffe in eine Spielhandlung, die die Entwicklung und damit die Inhaltsgebung verständlich macht, ein gangbarer Weg zu sein. Dies würde auch dem Anspruch der Visualisierung - dem Verständlichmachen eines Inhaltes mit den Mitteln, nicht aber „mit den Worten“ des Bildes - gerecht werden.

Bedenkt man den starken Einfluß der Bildmedien auf die Rezipienten, so ergibt sich daraus eine für die Kirchen lebenswichtige Notwendigkeit, diese Fragen zu erörtern und in Zusammenarbeit mit Medienfachleuten und Praktikern eine religiöse Bildsprache zu finden.

Aus der Betonung der Eigenständigkeit der Bildsprache zum besonderen Verständnis abstrakter Inhalte im Fernsehen ergibt sich die Forderung für eine optimale Visualisierung religiöser Inhalte:

\section{- die Eigenständigkeit der Bildkonzeption zur Textkonzeption}

Es geht darum, das Bild als die konstitutive Komponente der Totalsprache - definiert als Einheit von Bild, Wort, Geräusch und Musik - zu sehen. Peter Ruge sagt schon für den rein journalistischen Bereich: „Der Text ist nicht als Bildbeschreibung anzulegen, sondern ist Bildergänzung. Für den Fernsehjournalisten heißt das, daß er ein Thema optisch auflösen muß. Dieses Vorausdenken in Bildern führt auf die Suche nach dem Motiv, das sich als Träger einer Aussage eignet." 25

Daraus läßt sich für die Produktionen der FZ 3, die auch abstrakte und daher nur filmisch adäquat aufzuarbeitende Inhalte zu berücksichtigen hat, eine Motivüberlegung und Motivsuche als unbedingt notwendig ableiten. Weiter dürfte die Konzeption von Filmbeiträgen nur aus einem skizzenhaften Treatment, das jeweils starken Veränderungen ausgesetzt sein wird, und nicht aus einem vor Drehbeginn feststehenden und dann „nur mehr zu visualisierenden“ Text bestehen.

- die Einhaltung der filmdramaturgischen Gesetzmäßigkeiten und Verwendung aller formalen Möglichkeiten (z.B. Parallelmontage, Kontrastmontage etc.)

Für eine mediengerechte Visualisierung religiöser Inhalte sind - unter Einbeziehung aller in diesem Abschnitt „Problematisierung kirchlicher Verkündigung in den elektronischen Medien" aufgezeigten Faktoren - folgende Voraussetzungen maßgebend:

- Erarbeitung einer religiösen audio-visuellen Bildsprache, die der Wahrheit zu gesellschaftlicher Relevanz verhilft.

- Zusammenarbeit von Theologen (beratend) einerseits und Medienfachleuten andererseits, um einer filmischen Komposition Rechnung tragen zu können;

- weitgehender Verzicht auf die Selbstdarstellung der Kirchen und ihrer Aktivitäten zugunsten einer Darstellung jener Werte, für die die Kirchen eintreten - und das 
in medien- und rezipientengerechten Programmformen (Betonung der Spielformen);

- Erkennen der Probleme und Bedürfnisse der Rezipienten, denn: „Wenn eine Verkündigung zeitgemäß sein und dem konkreten Menschen, der immer in einer bestimmten geistigen und gesellschaftlichen Situation lebt, etwas sagen will, muß man zunächst wissen, was diese Menschen bewegt.“

\section{Zusammenfassung}

Ansätze zu einer theoretischen Erörterung der Medienarbeit innerhalb der Kirchen sind vorhanden. Die Frage, inwieweit Verkündigung im kommunikationswissenschaftlichen Sinne „Information“oder inwieweit sie Interpretation der Welt und der Schrift ist, bedürfte einer - auch für die Arbeit in den Massenmedien wichtigen umfassenden theologischen Auseinandersetzung. Erfreulich ist, daß sich die Kirchen zu einer Zusammenarbeit mit den Medienfachleuten und Praktikern in Hörfunk und Fernsehen bekennen. Ohne Zweifel sollte diese Arbeit intensiviert werden, will man zu besseren Resultaten kommen. Die Sendungen von der formalen Seite möglichst attraktiv zu gestalten, ist dabei ein wesentlicher Aspekt. Wichtiger scheint mir aber noch zu sein, in den Fragen nach dem Inhalt zu einer klaren Linie zu gelangen. Das entscheidende Problem besteht dabei darin, daß einerseits die Kirchen mit Recht für eine einheitliche inhaltliche Tendenz eintreten, daß andererseits ebenso zu Recht von den einzelnen Sprechern ein persönliches Zeugnis zu fordern ist, zwei Elemente also, die nicht leicht zu einer dauerhaften Übereinstimmung gebracht werden können. Die Kirchen müssen bestimmen, ob sie sich nicht doch mehr mit dem politischen Leben der Gesellschaft auseinandersetzen sollen, um damit den Erwartungen dieser Gesellschaft mehr entgegenzukommen.

$\mathrm{Da}$ sie sich fortwährend zu entscheiden habe, eine der effektiven Meinungsträger in dieser Gesellchaft zu sein, bringt eine Reihe von Konsequenzen mit sich: das Spektrum ihrer Aufgaben wird größer und damit ihre Verpflichtung, sich mit den Fragen der Öffentlichkeit fundiert auseinanderzusetzen. Aus dieser Verpflichtung ergeben sich für die mediale Verkündigung vielfältige Anforderungen. Können die Kirchen diese erfüllen, dann könnten sich die Sprecher - auf dieser verbreiterten Basis - als jeweils eine Stimme innerhalb der Kirche und nicht als die Stimme einer Institution verstehen. $\mathrm{Zu}$ Stellungnahmen zu den diversen Sachfragen würden Spezialisten herangezogen werden müssen, die aus christlicher Sicht diese Fragen interpretieren könnten.

Daraus ergibt sich aber auch für die Medien eine Konsequenz: die Kirchen nicht mehr in das Sonntags- oder Abseitsghetto zu sperren und sie als Mitarbeiter zu akzeptieren.

Die Kirchen wiederum können sich von ihrem Auftrag her gesehen in der Zusammenarbeit wohl als Partner der Medien verstehen; ihre wesentliche Aufgabe liegt aber darin, daß sie eine unsere Gesellschaft und damit auch indirekt den Rundfunk - verstanden als die Öffentlichkeitsarbeit der Gesellschaft - tragende Kraft darstellen sollen. Daraus resultiert die Verpflichtung einer verstärkten Mitarbeit in der Praxis, nicht nur in den kirchlichen Abteilungen und den Gremien des ORF, sondern an der breiten Basis des Unternehmens. 
Eine weitere Konsequenz für die Medien und für die Kirchen wird in der Konzeption inhaltlich und formal neuer Programmformen liegen müssen, um jene Mitglieder der Gesellschaft zu erreichen, die man bis jetzt noch nicht anzusprechen vermochte. Ein Umdenken unter Berücksichtigung der genannten Fakten wird die elektronischen Medien wie die Kirchen gleichermaßen betreffen. Es ist anzunehmen, daß dieses Umdenken, welches in und vor der Öffentlichkeit vor sich gehen müßte, sowohl den Kirchen als auch den Medien von der Öffentlichkeit honoriert werden würde.

Solange dieser Prozeß nicht initiiert ist, wird man die Feststellung des Hörfunk-Intendanten In der Maur zum 15jährigen Bestehen der Hauptabteilung Kirchenfunk hauptsächlich als eine Aufforderung zu dieser Entwicklung verstehen dürfen: „Der Kirchenfunk lebt und weiß um seine Bezüge zur Zeit und zur Sozietät. Solange er dies weiß und solange er sein Wissen - oft auch über Widersprüche hinweg programmlich verwirklicht, wird er stets ein wichtiger Programmbestandteil bleiben, meilenweit jenseits bloßer Pflichtübungen. Und das ist das Allerbeste, was man von ihm sagen und was man ihm wünschen kann."27

\section{Anmerkungen}

${ }^{1}$ Sie beruht auf der (theaterwissenschaftlichen) Dissertation der Autorin: Arten und Methoden der Kirchenfunksendungen der Gegenwart im österreichischen Rundfunk (Wien 1978).

2 Gespräch mit Rudolf Henz am 8.11.1973.

3 Punkt 5 wurde 1973 widerrufen.

4 Stand 1977 unter Generalintendant Otto Oberhammer.

5 Heinz Linnerz: Überlegungen zum Verhältnis Kirchen-Rundfunk, in: „medium“, 8. Jg., 1971, Heft 1, S. 26.

${ }^{6}$ Heinz Linnerz, a.a.O., S. 24.

7 Heinz Linnerz, a.a.O., S. 24.

8 Walter Schricker: Kirchliche Sendungen im Hörfunk, in: Die provozierte Kirche, hrsg. von Herbert Breit und Wolfgang Höhne, München 1968, S. 194.

9 Stefan Rehrl in dem Vortrag „Christliche Botschaft und ethische Imperative. Chancen der Gewissensbildung durch religiöse Sendungen in den Massenmedien“ anläßlich des „Seminar 75“ des Kath. Zentrums für Massenkommunikation in Salzburg, 1975.

10 Hans Jürgen Schultz: Information als Verkündigung? in: Die provozierte Kirche, a.a.O., S. 103.

11 Stefan Rehrl, a.a.O.

12 Bernhard Klaus: Massenmedien im Dienst der Kirche, Berlin 1970, S. 21.

13 Vgl. Lexikon der Psychologie, hrsg. von Arnold Eysenck und Meili, Freiburg i.Br. 1971, S. 215.

14 Walter Jens: Die Kanzelrede, hohe Kunst der Manipulation in: Manipulation in der Kirche?, hrsg. von Erhard Domay, Gütersloh 1977, S. 62.

15 Elmar Maria Lorey: Mechanismen religiöser Information, München 1970, S. 55.

16 Ebenda, S. $56 \mathrm{f}$. 
17 Ebenda, S. 57.

18 Durchgeführt von Dr. Fessel + GFK Gesellschaft für Konsum-, Markt- und Absatzforschung G.m.b.H. \& Co. KG.

19 Ebenda, Band D, S. 7.

20 Diese Untersuchung wurde unter dem Titel „Kirchliche Sendungen in Hörfunk und Fernsehen Juni/Juli 1972“ ebenfalls vom Institut Dr. Fessel, damals unter dem Titel: Dr. Fessel \& Co, Institut für Marktforschung, durchgeführt.

21 Hörfunkstudie 1976, Band D, Tabellenteil, S. $19 f$.

22 Hans Jürgen Schultz, a.a.O. S. 96.

23 Franz Zöchbauer: Verkündigung im Zeitalter der Massenmedien, München 1969, S. $41 \mathrm{f}$.

24 Hans-Dieter Bastian: Kommunikation, Stuttgart 1972, S. 153.

25 Peter Ruge: Praxis des Fernsehjournalismus, München 1975, S. 165.

26 Franz Zöchbauer, a.a.O., S. 45.

27 Wolf in der Maur: Der Hörfunk-Intendant zum Kirchenfunk-Jubiläum, in: „multimedia“, 4. Jg., Heft 13, 1976, S. 4.

\section{SUMMARY}

The basic work of the Austrian Radio's religious departments is treated here. The conditions under which broadcasts originate depend on historical, ecclesiastical, legal and organisational factors within the station itself. A future positive development of the religious departments depends not only on a better use of existing opportunities, but also on a new concept. To achieve this, a definition of information and proclamation is needed. Authors, too, must be freed from the necessity of acting on behalf of Church authorities. Listeners expect to hear comments and help on all aspects of daily life. Church broadcasting should aim at more than the mere transmission of theological knowledge; it should confront the world in a Christian spirit, leading to open dialogue. By closer involvement in all questions of public life, the Church would have a greater influence on society by its religious programmes.

\section{RÉSUMÉ}

Ce travail s'occupe des éléments fondamentaux du travail des services écclésastiques à la radio autrichienne. Les conditions selon lesquelles les émissions prennent naissance dépendent des occasions historiques, religieuses, juridiques et d'organisations internes. Cependant, il n'y a pas seulement un développement à venir positif des services écclésiastiques dans une meilleure utilisation des possibilités de réalisations, mais particulièrement dans un nouveau fondement:

Une définition de la relation entre l'information et la publication semble pour cela nécessaire; de même que les speakers écclésiastiques devraient être dispensés du devoir d'agir, directement comme représentants des autorités - c'est-à-dire des Eglises - . Les receveurs exigent des prises de position pour chaque question, souvent banale, de la vie quotidienne. Pour cela, le but des émission religieuses radio-télévisées ne doit pas seulement être la transmission d'un savoir théologique, mais la prise de position face au monde par une profession de foi qui aboutisse à un dialogue ouvert avec les receveurs. Par un engagement plus fort pour toutes les questions posées par la public, l'Eglise - et avec elle aussi les émissions religieuses à la radio pourrait à nouveau gagner plus d'importance dans notre société. 


\section{RESUMEN}

Este trabajo analize los elementos constitutivos de los programas eclesiales de la radio austriaca. Las características de cada emisión dependen de circunstancias históricas, eclesiales, jurídicas y de organización radiofónica. El desarrollo positivo de estos programas en el futuro depende no solo de una mejor utilización de las posibilidades de programación de la palabra; asímismo habría que librar a los portavoces de la Iglesia de la obligación de actuar como representantes de la autoridad - concretamente de la eclesiástica. Los receptores desean escuchar opinions sobre todos los problemas cotidianos, incluídos las más triviales. Por ello, la meta de la radiodifusión y de la televisión eclesial no puede ser la difusión de conocimientos teológicos, sino una toma de postura frente al mundo, basada en la convicción de que debe llegarse a un diálogo abierto con el receptor. Mediante un mayor compromiso respecto a todos los problemas planteados por la opinión pública, la Iglesia, y por ende también la radio eclesial, podía alcanzar nuevamente una mayor significación en nuestra sociedad. 